\title{
Fundamental Solution of Elliptic Equation with Positive Definite Matrix Coefficient
}

\author{
Khoirunisa' ${ }^{1}$, Corina Karim² \\ 1,2Department of Mathematics, Universitas Brawijaya
}

Email: khoir.n97@gmail.com,co_mathub@ub.ac.id

\begin{abstract}
We study the fundamental solution of elliptic equations with real constant coefficients

$$
\sum_{i=1}^{n} \sum_{j=1}^{n} a_{i j} D_{i}\left(D_{j} u\right)=0
$$

where $a_{i j}$ is a positive definite matrix. We obtained by searching the radial solution such that we solved the equation into ordinary differential equations.
\end{abstract}

Keywords: fundamental solution, elliptic equation, positive definite matrix

\section{INTRODUCTION}

We consider the linear differential operator

$$
L u=\sum_{i=1}^{n} \sum_{j=1}^{n} a_{i j}(x) \frac{\partial^{2} u}{\partial x_{i} \partial x_{j}}+\sum_{i=1}^{n} b_{i}(x) \frac{\partial u}{\partial x_{i}}+c(x) u,
$$

with coefficient defined in an $n$-dimensional domain $D$, where $b_{i}(x)$ and $c(x)$ are any functions that depend on $x$. $L$ is said to be of elliptic type (or elliptic) at a point $x^{0}$ if the matrix $a_{i j}\left(x^{0}\right)$ is positive definite, i.e., if for any real vector $\xi \neq 0, \sum_{i=1}^{n} \sum_{j=1}^{n} a_{i j} \xi_{i} \xi_{j}>0$ [3]. Laplace's equation $\Delta u=$ 0 is the simplest and most basic example of elliptic equation, and the Laplacian of $u$ is $\Delta u=$ $\sum_{i=1}^{n} u_{x_{i} x_{i}}$ [1]. While, a symmetric matrix $A$ is called a positive definite matrix if a quadratic form $x^{T} A x>0$ for all $x \neq 0, x \in \mathbb{R}[5]$.

A fundamental solution of the differential operator $L$ in $\Omega$ is a function $K(x, z)$ defined for $x \in \Omega, z \in \Omega, x \neq z$ and satisfying the following property: For any bounded domain $\mathbb{R}$ with smooth boundary $\partial \mathbb{R}$ and for any $z \in \mathbb{R}$,

$$
u(z)=\int_{\mathbb{R}} K(x, z) \overline{L^{*} \overline{u(x)}} d x,
$$

for every $u \in C_{0}^{m}(\mathbb{R})$, and $L^{*}$ is formal adjoint of $L[4]$.

Here, we define the following equation as elliptic equation with positive definite matrix

$$
\sum_{i=1}^{n} \sum_{j=1}^{n} a_{i j} D_{i}\left(D_{j} u\right)=0,
$$

where $a_{i j}$ is element of positive definite matrix, $u=u(x)$ and $x \in U, U \subset \mathbb{R}^{n}$. In 2016, Fitri studied the Holder regularity of weak solutions to linear elliptic partial differential equations with continuous coefficients, Campanato type estimates are obtained for the validity of regularity of solutions (see [2] ). In this paper, we study the fundamental solution of elliptic equation with positive definite matrix coefficient. Due to the symmetry of elliptic equation, radial solutions are natural to look for since the given partial differential equation can be reduced to an ordinary differential equation which is easier to solve. In this way, we can reduce the higher dimensional problems to one dimensional problems. 


\section{RESULTS AND DISCUSSION}

Let the elliptic equation with positive definite matrix as in (2) then to find a solutions $u$ of elliptic equations, it consequently seems advisable to search for radial solutions, that is functions of $r$.

Theorem 3.1 Let

$$
r=\sqrt{\sum_{i=1}^{n} \sum_{j=1}^{n} b_{i j} x_{i} x_{j}}
$$

where $b_{i j}$ is element of coffactor matrix $a_{i j}$. Then partial derivative of $r$ with respect to $x_{j}$ and partial derivative of $r$ with respect to $x_{i} x_{j}$ are defined by

and

$$
\frac{\partial r}{\partial x_{j}}=\frac{1}{r} \sum_{i=1}^{n} \sum_{j=1}^{n} b_{i j} x_{i}
$$

$$
\frac{\partial^{2} u}{\partial x_{i} \partial x_{j}}=\frac{1}{r} \sum_{i=1}^{n} \sum_{j=1}^{n} b_{i j}-\frac{1}{r^{3}} \sum_{i=1}^{n} \sum_{j=1}^{n}\left(\sum_{k=1}^{n} b_{i k} x_{k} \sum_{k=1}^{n} b_{j k} x_{k}\right) \text {. }
$$

Theorem 3.2 Suppose

$$
u(x)=v(r)
$$

then the second derivative of $u$ with respect to $x_{i} x_{j}$ denoted $D_{i}\left(D_{j} u\right)=\frac{\partial^{2} u}{\partial x_{i} \partial x_{j}}$ is the total derivative of $v(r)$ respect to $x_{i} x_{j}$.

Corollary 3.3 If the second derivative of (4) is substituted into the equation (2) then we get

$$
\begin{aligned}
\frac{v^{\prime \prime}(r)}{r^{2}} \sum_{i=1}^{n} \sum_{j=1}^{n} a_{i j} & \left(\sum_{k=1}^{n} b_{i k} x_{k} \sum_{k=1}^{n} b_{j k} x_{k}\right)+\frac{v^{\prime}(r)}{r} \sum_{i=1}^{n} \sum_{j=1}^{n} a_{i j} b_{i j} \\
& -\frac{v^{\prime}(r)}{r^{3}} \sum_{i=1}^{n} \sum_{j=1}^{n} a_{i j}\left(\sum_{k=1}^{n} b_{i k} x_{k} \sum_{k=1}^{n} b_{j k} x_{k}\right)=0 .
\end{aligned}
$$

Corollary 3.4 If $A$ is positive definite matrix with $a_{i j}$ element, and $B$ is cofactor matrix of $A$ with $b_{i j}$ element then

$$
I(\operatorname{det} A)=A B
$$

Corollary 3.5 If $A$ matrix and $B$ matrix are symmetric, then $\sum_{i=1}^{n} \sum_{j=1}^{n} a_{i j} b_{i j}$ can be simplified to be

$$
\sum_{i=1}^{n} \sum_{j=1}^{n} a_{i j} b_{i j}=n \operatorname{det} A
$$

Next, to simplify the sum of bracket in the first and the last terms in (5), we denoted $Y_{i}$ for $i=$ $1,2, \ldots, n$ and $j=1,2, \ldots, n$, so that we have

thus, the equation (5) can be rewrite as

$$
Y_{i}=(\operatorname{det} A) \sum_{i=1}^{n} \sum_{j=1}^{n} b_{i j} x_{i} x_{j}=(\operatorname{det} A) r^{2}
$$

then we have

$$
\frac{v^{\prime \prime}}{v^{\prime}}=\frac{1-n}{r}
$$




$$
v^{\prime}=a r^{1-n}
$$

Since, the domain $x$ is at $n$-dimension, $n \geq 2$ then $v(r)$ has the following general form:

$a, b, c \in \mathbb{R}$.

$$
v(r)=\left\{\begin{array}{lc}
a \log r+b, & n=2 \\
\frac{c}{r^{n-2}}+b, & n \geq 3 .
\end{array}\right.
$$

By rescaling (6) to $u(x)=v(r)$, where $r=\left(\sum_{i=1}^{n} \sum_{j=1}^{n} b_{i j} x_{i} x_{j}\right)^{\frac{1}{2}}$, then we get the fundamental solution of (2) is

where $a, b, c \in \mathbb{R}$.

$$
v(r)= \begin{cases}a \log \left(\sum_{i=1}^{n} \sum_{j=1}^{n} b_{i j} x_{i} x_{j}\right)^{\frac{1}{2}}+b, & n=2 \\ c\left(\sum_{i=1}^{n} \sum_{j=1}^{n} b_{i j} x_{i} x_{j}\right)^{\frac{2-n}{2}}+b, & n \geq 3\end{cases}
$$

\section{Remarks:}

We suppose the identity matrix $I_{n \times n}$ as a coefficient of (2). $I$ is a positive definite matrix, then the elliptic equation can be formed as follows

where

$$
\sum_{i=1}^{n} \sum_{j=1}^{n} a_{i j} D_{i}\left(D_{j} u\right)=0
$$

Then coffactor matrix

$$
a_{i j}= \begin{cases}1, & i=j \\ 0, & i \neq j\end{cases}
$$

$$
b_{i j}= \begin{cases}1, & i=j \\ 0, & i \neq j\end{cases}
$$

According to $a_{i j}$ and $b_{i j}$ as above, equation (7) can be written as

$$
\sum_{i=1}^{n} D_{i}\left(D_{i} u\right)=0
$$

where (8) is equivalent to the Laplace's equation $\sum_{i=1}^{n} u_{x_{i} x_{i}}$.

\section{REFERENCES}

[1] Evans, L. C. (1998). Partial Differential Equation. Second Edition. American Mathematical Society: USA

[2] Fitri, S. (2016). Campanato Type Estimates for Solutions of an Elliptic Systems Class. International Interdisciplinary Studies Seminar Proceeding. 12-17. Malang: Universitas Brawijaya.

[3] Friedman, A. (1964). Partial Differential Equation of Parbolic Type. Dover Publication,Inc: New York.

[4] Friedman, A. (1969). Partial Differential Equation. Dover Publication,Inc: New York.

[5] Leon, S. J. (2001). Aljabar Linear dan Aplikasinya. Erlangga: Jakarta. 